\title{
Tuberculosis of the Breast: A Challenging Component of Benign Breast Diseases
}

\author{
Rajbangshi M.C. ${ }^{1}$, Deka Sumi ${ }^{2}$, Mahanta Bandana ${ }^{3}$, Mahanta Putul ${ }^{4}$ \\ ${ }^{1}$ Associate Professor, Department of Surgery, Gauhati Medical College and Hospital, Guwahati, Assam, India, \\ ${ }^{2}$ Demonstrator of Biochemistry, Gauhati Medical College and Hospital, Guwahati, Assam, India, ${ }^{3}$ Associate \\ Professor, Department of Anaesthesiology, Tezpur Medical College and Hospital, Tezpur, Assam, India, ${ }^{4}$ Professor, \\ Forensic Medicine and Toxicology
}

\begin{abstract}
Introduction: Tuberculosis (TB) of the breast is a rare manifestation of extra-pulmonary localization of the disease. The incidences vary from a minimum in developed countries to a high in developing countries like India and Africa.
\end{abstract}

Materials and Method: This study was a hospital-based observational study carried out in 75 cases of benign breast disease which were studied with special reference to Tuberculosis of the breast. All patients who were clinically diagnosed as a benign breast lump, above the age of 14-years and among them who were willing to undergo investigations and treatment were included. Ziehl-Neelsen staining was performed in all fine needle aspirates and finally, treatment modality used.

Results: The present study involved 67 cases of benign breast lumps and 8 cases of recurrent breast abscesses. Further, it depicts predominant benign breast lumps which were fibroadenoma in $65.7 \%$ fibroadenosis in $23.80 \%$, TB breast in $4.50 \%$, ductal papilloma in $2.90 \%$ and phyllodes tumour of the breast in $1.5 \%$ of cases.

Conclusion: For the TB of the breast in India, various treatment modalities are available. With timely diagnosis, the morbidity can be minimised with less amount of suffering.

Keywords: Breast diseases; Ziehl-Neelsen staining, fine needle aspiration cytology (FNAC).

\section{Introduction}

Tuberculosis (TB) of the breast is a rare manifestation of extra-pulmonary localization of the disease which accounts for less than $0.1 \%$ of breast conditions in developed countries but reaches $3-4 \%$ in regions where the disease presents with high incidence (India, Africa). ${ }^{1}$ It appears mostly in women of reproductive age, multiparous, lactating. It has been scarcely reported to infect male patients, mainly before puberty, as well as women of older age. The most common presentation is that

\section{Corresponding Author:}

Mahanta Putul

Professor, Forensic Medicine and Toxicology

e-mail: drpmahanta@gmail.com

Mobile: +9435017802 of a tumour in the middle or upper-outer quadrant of the breast, with multifocal involvement being rarely documented. The differential diagnosis includes breast cancer and abscess formation. The first case of breast tuberculosis was recorded by Sir Astley Cooper who described it as "scrofulous swelling of the bosom". Tuberculosis is caused by Mycobacterium tuberculosis and affects primarily the lungs. Breast tuberculosis is a rare disease, with an incidence of less than $0.1 \%$ of all breast lesions in Western countries and 3-4\% in tuberculosis endemic regions, such as India and Africa. It usually affects young lactating multiparous women, although it may also be reported in prepubescent males or elderly women. ${ }^{2,3}$ Most commonly, the disease presents as a lump in the central or upper-outer quadrant of the breast, while multiple lumps appear less frequently ${ }^{4}$ The clinician may misdiagnose breast tuberculosis with either breast carcinoma or abscess. ${ }^{4,5}$ Diagnosis 
of breast tuberculosis, therefore, remains a challenge for clinicians and requires a high degree of suspicion. Mammography or ultrasonography are unreliable in distinguishing the breast tuberculosis from carcinoma because of the variable pattern of presentation of such inflammatory lesion. Histopathology plays a pivotal role in the diagnosis. Surgery is reserved only for selected refractory cases. ${ }^{3}$

This paper aims to diagnose tuberculosis of breast and provide appropriate treatment.

\section{Materials and Method}

The present study deals with 75 patients of benign breast diseases with special reference to $\mathrm{TB}$ of the breast. The data were collected from the six surgical units of Gauhati Medical College and Hospital, Assam, during the period from $1^{\text {st }}$ July 2017 to $30^{\text {th }}$ June 2018 . All patients who were clinically diagnosed as a benign breast lump, above the age of 14-years and among them who were willing to undergo investigations and treatment were included. Ziehl-Neelsen staining was performed in all fine-needle aspirates.

Evaluation of the cases was done by a thorough clinical breast examination. The breasts are assessed for nodularity and presence of any dominant mass or thickening. The next step was to palpate the regional lymph nodes. These include the supraclavicular, infraclavicular and axillary nodes. Fine needle aspiration cytology (FNAC) was done (Harris Alum - Haematoxylin, Orange G 6 and EA 36 solutions for staining). Ziehl-Neelsen staining to stain mycobacterium tuberculosis and mycobacterium leprae was performed. Ultrasonography of the benign breast tumours was also performed to locate the smooth contours. Round or oval shapes, with weak internal echoes and well defined anterior and posterior margins. Cysts on ultrasound examination, are always well-circumscribed, with smooth margins and have an echo-free centre irrespective of the sensitivity settings. Diagnostic mammography was performed in case of breast abnormality on clinical examination or screening mammography. Calcifications were assessed by it as micro-calcifications and macrocalcifications, mass or cyst is another important change seen on mammograms (non-cancerous, fluid-filled sacs, fibroadenomas). The biopsy was done in all where mammography shows abnormal results. Before collection of the data ethical approval was taken from the ethics committee.

\section{Results and Observations}

The present study diagnosed 67 cases of the benign breast lump and 8 cases of recurrent breast abscess who attended outpatient department as well those who were admitted in the six surgical units of Gauhati Medical College \& Hospital, Guwahati during the period of $1^{\text {st }}$ July 2017 to $30^{\text {th }}$ June 2018 . The data collected from 67 cases are studied based on etiological and clinical backgrounds, the accuracy of diagnostic investigations and finally treatment modality used. During this study, among the patients with a breast lump, only those cases were evaluated who on examination appeared benign and whose ultrasonographic features and FNAC results were benign have been included. All the cases who underwent surgery operated specimen were sent for histopathology.

Table 1: Incidence of the benign breast lump

\begin{tabular}{|l|c|c|}
\hline Type & No. of Cases & Percentage \\
\hline Fibroadenoma & 44 & $65.7 \%$ \\
\hline Fibro adenosis & 16 & $23.8 \%$ \\
\hline TB breast & 3 & $4.5 \%$ \\
\hline Duct papilloma & 2 & $2.9 \%$ \\
\hline Phyllodes tumour & 1 & $1.5 \%$ \\
\hline Duct ectasia & 1 & $1.5 \%$ \\
\hline
\end{tabular}

Table 1 depicts among 67 cases presented with a palpable lump, among them 44 (65.7\%) were diagnosed to be a fibroadenoma, followed by fibro adenosis (23.8\%), TB breast (4.5\%), Ductal papilloma (2.9\%), duct ectasia and phyllodes (1.5\% each).

Bar diagram depicts four cases of tuberculosis of the breast were diagnosed, among those three presented as painful lump while others presented as an abscess. 8 cases of recurrent abscesses were included in this study. Pus was sent for Ziehl-Neelsen staining, Bacterial culture and sensitivity and AFB culture. Although Ziehl-Neelsen staining was negative in all of them, AFB culture identified Mycobacterium Tuberculosis, hence the diagnosis of Tuberculosis of the breast was made. 


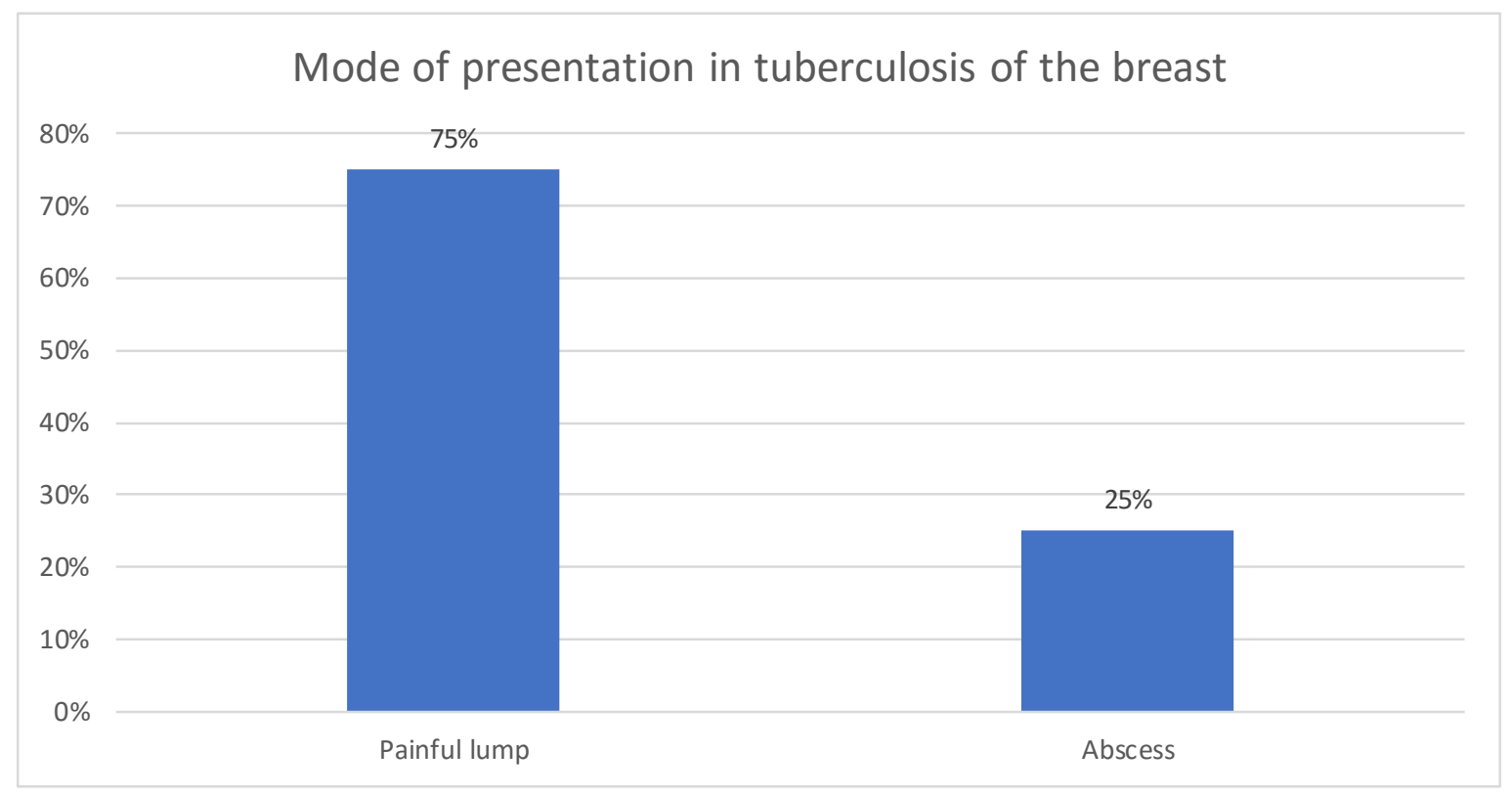

Figure 1 Bar diagram showing modes of presentation in tuberculosis of the breast

Table 2: Significance of Ziehl-Neelsen staining in tubercular mastitis

\begin{tabular}{|l|c|c|}
\hline Ziehl-Neelsen staining & No. of Cases & Percentage \\
\hline Positive & 0 & $0 \%$ \\
\hline Negative & 4 & $100 \%$ \\
\hline
\end{tabular}

Table 2 depicts Four cases of tubercular mastitis were diagnosed, three of them presented as lumps while one presented as an abscess. All of them stained negative for Ziehl-Neelsen, however, lumps were histopathologically diagnosed as tuberculosis of the breast. While abscess was empirically started on ATT due to recurrent history. One case of fibroadenoma also stained positive for Ziehl-Neelsen, however, it was histopathologically diagnosed postoperatively to be fibroadenoma and was a false positive.

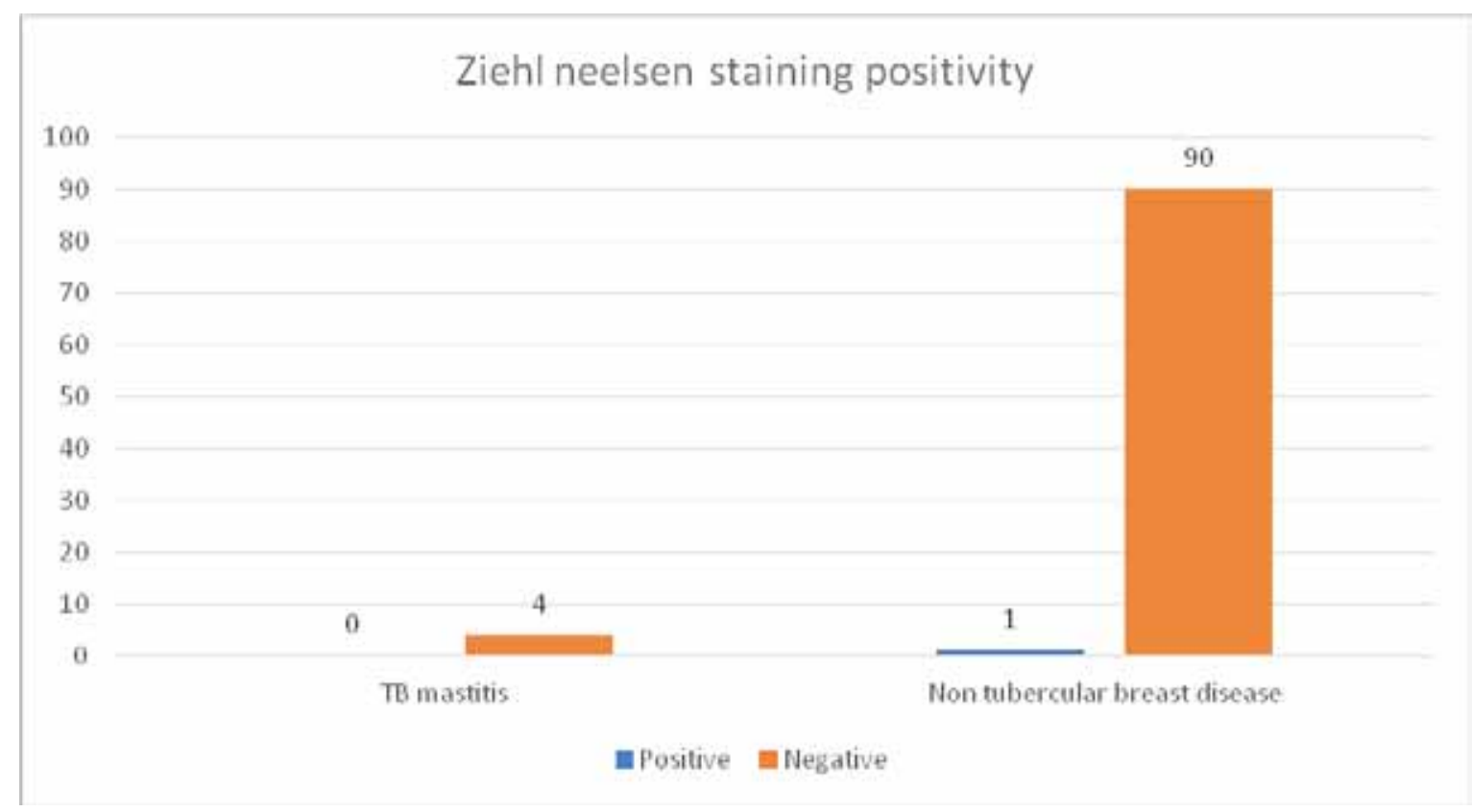

Figure 2 Bar diagram showing the significance of Ziehl-Neelsen staining in the diagnosis of tuberculosis of the breast 
Figure 2 describes Tuberculous mastitis (TM) occurs far more frequently in women, especially in their reproductive age, and is uncommon in prepubescent and elderly women.

\section{Treatment Modalities:}

Tuberculosis of the Breast: In this study 4 cases of tuberculosis of breast were diagnosed. 3 of them presented as lumps and one as an abscess. Lumps were excised due to initial diagnostic uncertainty and abscess was drained by open drainage. Pus was sent for both Ziehl-Neelsen staining and AFB culture. Although Ziehl-Neelsen came out to be negative, AFB culture identified Mycobacterium Tuberculosis. All were given 9 months of antitubercular therapy with 2 months of intensive therapy with ethambutol (E) 1200 $\mathrm{mg}$; rifampicin (R) $450 \mathrm{mg}$, isoniazid $(\mathrm{H}) 600 \mathrm{mg}$ and pyrazinamide (Z) $1500 \mathrm{mg}$., followed by 7 months of ethambutol and isoniazid. Improvement was observed during our period of study, however, long term follows up was not possible.

\section{Discussion}

The relative incidence of various benign breast lumps in were found to be concurrent with other reported series of Sangma et al., ${ }^{6}$ Seema et al., ${ }^{7}$ Amruthavalli et al., ${ }^{8}$ Jagtap et al., ${ }^{9}$ and Abhijit MG et al. ${ }^{10}$

Significance of Ziehl-Neelsen staining in tuberculosis of breast: Four cases of tubercular mastitis were diagnosed, three of them presented as lumps while one presented as an abscess. All the lumps stained negative for Ziehl-Neelsen; however, it was histopathologically diagnosed as tubercular mastitis. Abscess aspirate also stained negative for Ziehl-Neelsen. Positivity of Ziehl-Neelsen staining was found to be statistically insignificant. This finding was consistent with the study by Gupta PP et al. ${ }^{11}$ On all 7 patients of tuberculosis of breast in which the positive Ziehl-Neelsen stain was only in $38 \%$, and was found to be statistically insignificant for the diagnosis.

Presentation of tuberculosis of breast: Among four patients diagnosed with tuberculosis of breast, $3(75 \%)$ of them presented with a lump and $1(25 \%)$ presented as an abscess. Finding was consistent with the study by Mallika Tewari and HS Shukla ${ }^{12}$ on 30 patients with Tuberculosis of the breast in which $73 \%$ presented with lump and $27 \%$ with abscess.

Tuberculous mastitis (TM) occurs far more frequently in women, especially in their reproductive age, and is uncommon in prepubescent and elderly women. ${ }^{13}$ This parallels the highest incidence of pulmonary tuberculosis. This could be because the female breast undergoes frequent changes during the period of childbearing activity and is more susceptible to trauma and infection. ${ }^{14}$

\section{Treatment Modalities:}

Tuberculosis of the Breast: Our treatment protocol was similar to that followed by Mallika Tewari and HS Shukla. ${ }^{12}$ However the protocol followed by Prem Prakash et al., ${ }^{11}$ was of 6 months ATT and observed good response rate in $96 \%$ of the cases. In our study, there were only 2 cases of ductal papilloma and single case each of phyllodes tumour and ductal papilloma. Simple mastectomy was performed for the phyllodes tumour and microdiscectomy for ductal papilloma and duct ectasia. Wide excision is usually sufficient for phyllodes tumour however in this case size was large enough to make wide excision improbable. Techniques we followed were similar to the technique followed by Akshara et al., ${ }^{15}$ and Naveen et al., ${ }^{16}$ in their studies on benign breast disease for these lesions.

In the era of minimally invasive surgery, percutaneous drainage has proven a safe and effective alternative to incision and drainage in acute abscesses, while chronic and recurrent abscesses are best treated with the classical means of drainage. The method of percutaneous aspiration combined with irrigation and installation of antibiotics has proved effective in $96 \%$ of cases reported in one series. ${ }^{17}$ In this current study, only patients with recurrent breast abscess were included. Hence, we performed emergency incision and drainage and pus was sent for culture and sensitivity. Our management method was similar to that followed by different studies on benign breast diseases. ${ }^{18,19}$

\section{Conclusion}

Benign breast diseases are essential to differentiate from malignant ones and also between one another. An appropriate diagnosis is key to have a proper outcome.

Conflict of Interest: None declared.

Ethical Clearance: Taken.

Source of Funding: None declared 
Author Disclosure: The article is original with the author(s) and does not infringe any copyright or violate any other right of any third party. The article has not been published (whole or in part) elsewhere and is not being considered for publication elsewhere in any form, except as provided herein. The first author has conceived the study design and helped in the collection of data along with author second. The third and fourth author has helped in interpreting the data thus collected and also in writing the manuscript along with the author one and second. All author(s) have contributed sufficiently in the article to take public responsibility for it and have reviewed the final version of the manuscript and approved it for publication.

\section{References}

1. Cooper SA. Illustrations of the diseases of the breast Part 1. London: Longmans, Rees, Brown Orme, Green, and Longman; 1829. p. 7

2. Luh SP, Chang KJ, Cheng JH, Hsu JD, Huang CS. Surgical treatment for primary mammary tuberculosis - report of three octogenarian cases and review of literature. Breast Journal 2008;14:311312.

3. Maroulis I, Spyropoulos C, Zolota V, Tzorakoleftherakis E. Mammary tuberculosis mimicking breast cancer: a case report. Journal of Medicine Case Reports 2008;2:34.

4. Mirsaeidi SM, Masjedi MR, Mansouri SD, Velayati AA. Tuberculosis of the breast: report of 4 clinical cases and literature review. East Mediterranean Health Journal 2007;13:670-676.

5. Sriram KB, Moffatt D, Stapledon R. Tuberculosis infection of the breast mistaken for granulomatous mastitis: a case report. Cases J 2008 Oct 25;1:273.

6. Sangma MB Maychet, Panda Kishori, Dasiah Simon. (2013). A clinico-pathological study on benign breast diseases. J clin Diagn Res 2013 Mar;7(3):503-506.

7. Seema Nagger, Irbinder Kour Bali, Prachi Arun, Vinay Kumar, Kalpana Goyal, Parveen Shah, et al. Clinico-pathological study on benign breast diseases in Gurugram NCR. IOSR Journal of Dental and Medical Sciences (IOSR-JDMS) 2018 April;17(4):59-62.
8. BV Amruthavalli, V Srihari. Clinical study of benign breast diseases. IOSR Journal of Dental and Medical Sciences (IOSR-JDMS) 2015 Nov;14(11):34-40.

9. Jagtap SV, Nihalani RR, Boral S. Clinicocytopathological study of breast lump in the non-malignant categories. Asian Pac J Health Sci 2018;5(1):107-110.

10. Abhijit MG, Anantharaman D, Sumanth B, Ranjani R. Benign breast diseases: experience at a teaching hospital in rural India. Int J Res Med Sci 2013;1:738.

11. Gupta PP, Gupta KB, Yadav RK, Agarwal D. Tuberculous mastitis: A review of seven consecutive cases. Indian J Tub 2003;50:47-50.

12. Tewari M, Shukla HS. Breast tuberculosis: Diagnosis, clinical features and management. Indian J Med Res 2005;122:103-10.

13. Jalali U, Rasul S, Khan A, Baig N, Khan A, Akhter R. Tuberculous mastitis. J Coll Physicians Surg Pak 2005;15:234-7.

14. Raw N. Tuberculosis of the breast. Br Med J 1924;J:657-8.

15. Gupta A, Gupta AK, Goyal R, Sharma K. A study of clinical profile of benign breast diseases presenting at a tertiary care centre in central India. Scholar J Appl Med Sci 2015;3(2C):695-700.

16. Gupta A, Gupta AK, Goyal R, Sharma K. A study of clinical profile of benign breast diseases presenting at a tertiary care centre in central India. Scholar J Appl Med Sci 2015;3(2C):695-700.

17. Naveen N, Avijeet $M$ and Vikrant M.A clinical study of benign breast disease in rural population. Journal of Evolution of Medical and Dental Sciences 2013;12:5499-511.

18. Leborgne F. Treatment of breast abscesses with sonographically guided aspiration, irrigation, and instillation of antibiotics. AJR 2003;181(4):108991.

19. Abdel Hadi, Maha Bukhari, Huda. Breast infections in non-lactating women. Journal of family \& community medicine 2005;12:133-7. 